\section{AL-AZHAR}

Assiut Dental Journal
The Official Publication of The

Faculty of Dental medicine.

Al-Azhar Assiut Uniuersity.

Egypt

\title{
Evaluation of TMJ Functions after conservative Treatment of Unilateral Subcondylar Fracture
}

\author{
Mohammed Mahgob Mohammed Al-Ashmawy"1
}

Codex : 09/1901

Aadj@azhar.edu.eg

\section{KEYWORDS}

Displacement, Nonsurgical ,TMJ, Subcondylar fracture,

1. Department of Oral and Maxillofacial Surgery, Faculty of Dental Medicine, Al-Azhar University ,Assiut.

* Corresponding author e-mail: mohamedelashmawy.19@ azhar.edu.eg

\begin{abstract}
Aim: It is to assess TMJ functions after conservative treatment of unilateral subcondylar fractures. Subjects and Methods: A prospective study was conducted on 20 patients with unilateral mandibular subcondylar fracture undergoing nonsurgical treatment. Clinical and radiographic examinations were done prior to treatment and at 12-month follow up. Pain, perceived occlusion, mouth opening, protrusion, and horizontal movements of the mandible were evaluated by clinical examination. Radiologic evaluation was done using panoramic radiographs. Results: At 12-month follow-up, there was minimal pain in the temporomandibular joint region, there was an improvement in the perceived occlusion, without limited mouth opening. There was insignificant absolute difference between left and right lateral mandibular movements. The amount of increase in the protrusion of mandible was insignificant. On radiographic evaluation, mean ramus height pretreatment and 12 months post -treatment were $1.51+0.45$ and $1.47+0.48$, respectively. Conclusion: Unilateral subcondylar fractures of the mandible can be treated nonsurgically in patients with minimal occlusal discrepancies, adequate mouth opening, minimal displacement of condyle, and minimal ramus height shortening.
\end{abstract}

\section{INTRODUCTION}

According to various studies, mandible is the frequently involved bone among of all facial injuries. Mandible due to its prominent position is often involved in maxillofacial trauma, contributing to about 65-70\%. Therefore, mandibular fracture stands first when compared to zygomatic and maxillary fracture by a ratio of 6:2:1 $1^{1,2}$. Most common site of fracture in the mandible is the condylar process which accounts for $17.5-52 \%$.

This type of fracture usually occurs by an indirect blow to the other regions of the mandible. It is also seen in association with other mandibular fractures. Since decades, management of condylar fracture remains controversial whether to be treated conservatively or surgically ${ }^{3}$. 
Treatment of fractures of the condyle depends on many factors including clinical and radiological evidence for the presence of the fracture, extent (whether unilateral or bilateral), level of the fracture, degree of displacement or dislocation, the presence of additional facial fractures, deranged occlusion and mandibular dysfunction, posterior occlusal support, clinical experience of the surgeon, and willingness of the patient to undergo surgery ${ }^{4-6}$.

As in literature, three main treatments are advocated for adult condylar fractures: 1. Closed reduction with maxillomandibular fixation $[\mathrm{MMF}]$ followed by functional rehabilitation. 2. Functional therapy without MMF. 3. Open reduction with / without maxillomandibular fixation [MMF]. In the first two treatments, surgical procedure on the fractured segments is not undertaken and hence is form of closed treatment ${ }^{7}$. Recently, endoscopically assisted intraoral approach is showing promising results $^{8,9}$

Closed treatment involving intermaxillary fixation, followed by active physical therapy, had been mainly used to avoid problems following surgical approach such as facial never injury, skin scar, infection... etc ${ }^{10-12}$. Accordingly, this study was to evaluate TMJ function after nonsurgical treatment of unilateral mandibular subcondylar fractures presenting with minimal dental malocclusion.

\section{SUBJECTS AND METHODS}

The present study was done on patients with unilateral mandibular condyle fractures and who underwent nonsurgical treatment for the fracture from 2014 to 2018. The study patients were obtained from the Out-patient Clinic of Oral and Maxillofacial Surgery Department, Faculty of Dentistry, Al-Azhar university-Assiut branch

\section{Inclusion Criteria}

- Subcondylar fracture of mandible less than 1 week old demonstrated on roentgenograms

- Malocclusion
- $\quad$ Limited mouth opening

\section{Exclusion Criteria}

- Patients with history of any psychiatric disorders or mental retardation

- Patients who had mandibular function impairment or (TMJ pain or pain in the muscles of mastication prior to fracturing the mandibular condyle.

Treatment of the fractured mandibular condyle was performed according to the standard procedures of the department. Any other associated fractures of mandible if present were treated by open reduction and internal fixation. Arch bars were used to stabilize such fractures preoperatively and were later used for intraoperative MMF. The arch bars were also utilized for placement of guiding elastics postoperatively to treat the condylar fracture. Patients having subcondylar fractures with dental malocclusion without any other associated mandibular fractures were treated with arch bars and guiding elastics for occlusion repair. The rigid intermaxillary fixation was not used. Physiotherapy and continuous rehabilitation to reach to the normal ranges of jaw movements were continued for three months postoperatively.

\section{Postoperative evaluation:}

A prospective study was done on patients with fracture of the unilateral mandibular subcondyle treated nonsurgically. All the patients included in the study were clinically assessed for mandibular function and were radiographically evaluated for the displacement of the fractured mandibular condyle. Patients were recalled to the department 3,6,9,12 months postoperatively for follow-up.

\section{a- Clinical evaluation}

At follow-up, physical examination was performed by one examiner. Maximal mouth opening, left and right lateral mandibular movements and protrusion were measured with a measuring scale. 
Maximal inter-incisal distance (mouth opening) was taken as vertical range of motion left lateral movement, right lateral movement, and protrusion were taken as horizontal range of movements and were measured as the movement of the mandibular central incisors relative to the maxillary central incisors in the horizontal plane.

Visual analog scale (VAS) of $100 \mathrm{~mm}$ was used to measure the average pain intensity experienced by the patient pretreatment and during the week prior to follow-up. The VAS is a line with "no pain" at one end and "worst imaginable pain" at the other end. Objective analysis of occlusion was done as part of routine intraoral examination of the department and was noted as either stable or deranged. Perceived occlusion was assessed, by asking the patients whether they rated their occlusion good, moderate, or poor.

\section{b-Radiographic Evaluation:}

Panoramic radiographs were done preopertively 3,6,9,12 months postoperatively. Sagittal displacement of the fractured condyle and vertical overlap of the fractured condylar process fragment were measured using panoramic radiograph. Assessments of pretreatment and 12 months after nonsurgical treatment were used (fig,1).

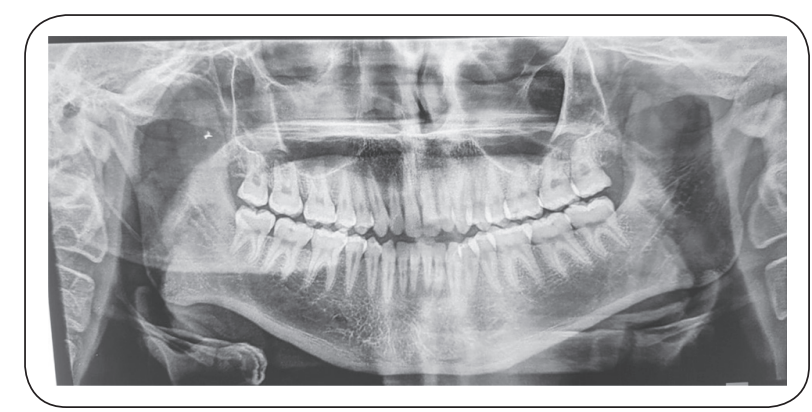

Fig. (1) Preoperative OPG for right unilateral subcondylar fracture.

\section{Statistical analysis}

The collected data were entered into the Microsoft excel sheet and were subjected to further statistical analysis to assess the mandibular function after nonsurgical treatment of mandibular condyle. All data were presented as mean and standard deviation and categorical data as numbers and percentages. Unpaired Student t-test was used for quantitative analysis and Chi-square test was used to test association. The statistical analysis was performed using the SPSS package (statistical package for social sciences version 17).

\section{RESULTS}

This study included twenty patients with ages ranging from 20 to 40 years with an average of 20 years. 12 males and 8 females were assigned to the current study. All patients were presented with unilateral subcondylar fractures. 15 patients were presented with right subcondylar fractures and 5 patients showed left subcondylar fractures. Fracture healing was satisfactory in all patients with clinical and radiographical evidence of union of fracture segments at the end of 12 months.

\section{Pain level evaluation:}

Table 1 shows the mean SD and frequency of VAS recorded pretreatment and 12 months posttreatment. There was mild pain in the TMJ region after mandibular condyle fracture in $60 \%$ of cases. While, at 12 months post-treatment, there was minimal pain in the TMJ region after closed treatment of the fracture in $10 \%$ of cases. There was a significant difference between means value of pretreatment and 12 month postoperative.

Table (1) Mean \pm SD and frequency of visual analog scale recorded at 6 and 12 month interval

\begin{tabular}{|c|c|c|c|c|}
\hline \multirow{2}{*}{ Variable } & \multicolumn{2}{|c|}{ Mean \pm SD } & \multicolumn{2}{c|}{ Frequency } \\
\cline { 2 - 5 } & Pretreatment & 12 month & Pretreatment & 12 month \\
\hline $\begin{array}{c}\text { VAS } \\
\text { values }\end{array}$ & $7.6+2.5$ & $2+1.5$ & $60 \%$ & $10 \%$ \\
\hline
\end{tabular}




\section{Occlusal state evaluation:}

Table 2 shows the occlusion perceived by the patient. There was improvement in perceived occlusion in some patients as only two patient perceived the occlusion as poor at 12 months (fig. $2 \mathrm{a}$ ).

Table (2) Perceived occlusion

\begin{tabular}{|c|c|c|c|}
\hline $\begin{array}{c}\text { Perceived } \\
\text { occlusion }\end{array}$ & Good & Moderate & Poor \\
\hline Pretreatment & $5(25 \%)$ & $8(40 \%)$ & $7(35 \%)$ \\
\hline At 12 month & $14(70 \%)$ & $3(20 \%)$ & $2(10 \%)$ \\
\hline
\end{tabular}

\section{Mouth opening :}

Preoperatively 9 patients had mouth opening of $<26 \mathrm{~mm}, 11$ patients had mouth opening of $26-41$ $\mathrm{mm}$. At 12 month postoperatively, all patient had reached to normal range of mouth opening more than $42.5 \mathrm{~mm}$ with mean value $49.1 \mathrm{~mm}$.

Tables 3 and figure 2 (b) shows the physical examination of the range of motion of mandible. In this study, the improvement in mouth opening and other movements were significant.

Table (3) Range of mandibular movements

\begin{tabular}{|c|c|c|c|c|}
\hline \multirow{2}{*}{ Variable } & \multicolumn{2}{|c|}{ Mean \pm SD } & \multirow{2}{*}{$\begin{array}{c}\mathrm{T} \\
\text { value }\end{array}$} & \multirow{2}{*}{$P$ value } \\
\hline & Pretreatment & 12 month & & \\
\hline $\begin{array}{l}\text { Maximum } \\
\text { mouth } \\
\text { opening }\end{array}$ & $23.73 \pm 7.30$ & $41.20 \pm 7.76$ & 0.471 & 0.640 \\
\hline $\begin{array}{l}\text { Left lateral } \\
\text { excursion }\end{array}$ & $3.33 \pm 3.67$ & $7.23 \pm 3.46$ & 0.108 & 0.914 \\
\hline $\begin{array}{l}\text { Right lateral } \\
\text { excursion }\end{array}$ & $4.13 \pm 3.38$ & $7.20 \pm 3.30$ & 0.077 & 0.939 \\
\hline Protrusion & $3.46 \pm 3.11$ & $6.53 \pm 3.20$ & 0.082 & 0.939 \\
\hline
\end{tabular}

\section{Displacement of fractured condyle}

Table 4 shows the displacement of condylar process fracture assessed by panoramic view. There are insignificant differences between both follow up intervals in ramus height difference and condyle / ramus difference (fig.3).

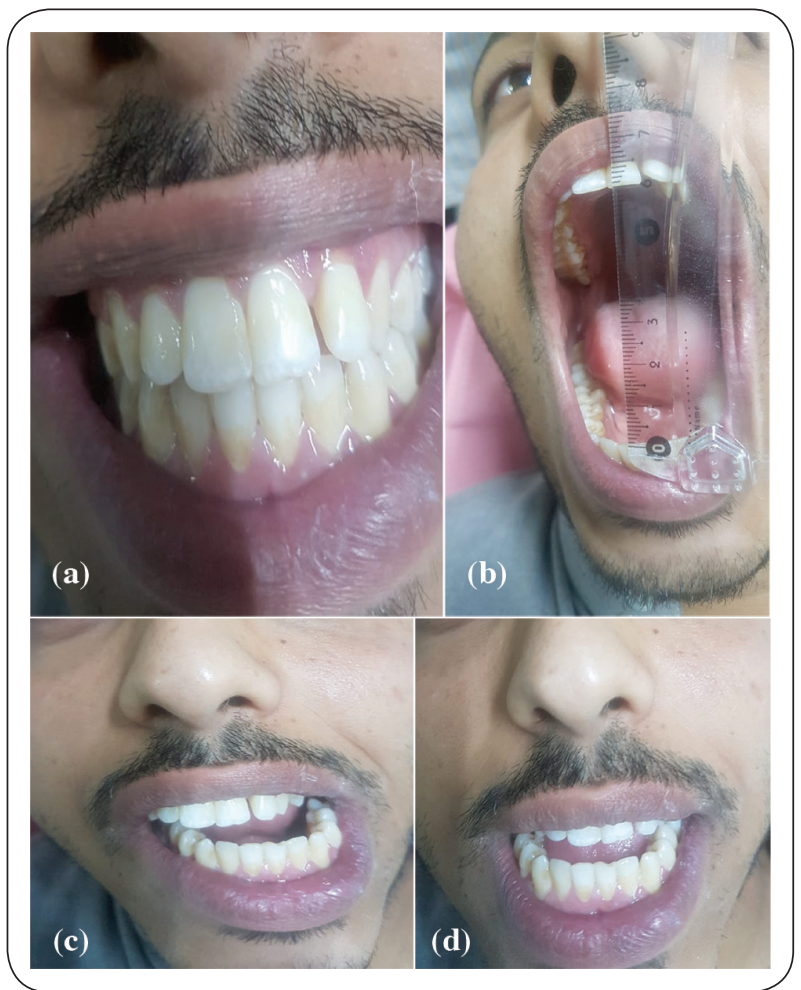

Fig. (2) a) proper occlusion at 12 month b) maximum opening at 12 month c) free lateral movement at 12 month d) free protrusion movement at 12 month

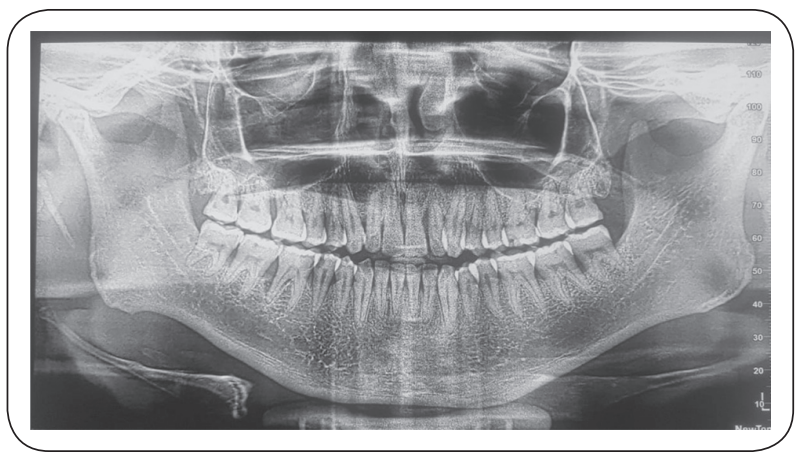

Fig. (3) Post-treatment OPG at 12 month

Table (4) Displacement of fractured condyle

\begin{tabular}{|c|c|c|c|c|}
\hline \multirow{2}{*}{ Variable } & \multicolumn{2}{|c|}{ Mean \pm SD } & T value & P value \\
\cline { 2 - 3 } & Pretreatment & 12 month & & \\
\hline $\begin{array}{c}\text { Sagittal plane } \\
\text { (panoramic image): } \\
\text { Condyle/ramus } \\
\text { angle difference }\end{array}$ & $4.72 \pm 1.9$ & $4.69 \pm 2.1$ & 0.0000 & 1.020 \\
\hline $\begin{array}{c}\text { Ramus height } \\
\text { difference } \\
\text { (panoramic image) } \\
(\mathrm{mm})\end{array}$ & $1.51 \pm 0.45$ & $1.47 \pm 0.48$ & 0.796 & 0.451 \\
\hline
\end{tabular}




\section{DISCUSSION}

The management of condylar fracture has generated more discussion and controversy. Conservative method of treatment was favored than surgical treatment. Advantages of conservative treatment includes the avoidance of hospitalization, general anesthesia, and surgery related complication like scarring, infection, and additional trauma, nerve injuries, and resorption of the condyle after reduction and fixation as a free graft $^{10-12}$. However, the limitations of closed reduction have also been extensively discussed. Hence, several studies concluded that patient treated by closed reduction had greater incidence of malocclusion, mandibular asymmetry, impaired masticatory function and pain localized to the affected joint and masticatory muscles ${ }^{13-15}$.

Walker $^{15}$ in 1994 discussed the goals for management of condylar fractures. These included pain free movement of the mandible, good occlusion, symmetry of the mandible and good facial jaw symmetry. He stated that the protocol in the management of mandibular subcondylar fractures is to achieve those goals irrespective of the type of management.

Current study was a trial to assess conservative treatment in nondisplaced subcondylar fractures. At 12 months post-treatment, VAS values presented minimal or no pain in the TMJ region after closed treatment of condylar fracture in the present study. Following trauma, there may be a varying degree of limited mandibular movements due to muscle spasm, edema, and hemarthrosis. These factors predispose to mandibular deviation to the injured side on mouth opening. This matched with Santler et $\mathrm{al}^{16}$ and MacLennan et $\mathrm{al}^{17}$.

Good perceived occlusion could be because of reduced differences between the ramus height on the fractured and non-fractured sides, which prevents premature occlusal contacts of posterior teeth on the fractured side. This is as the same in Niezen et al ${ }^{18}$ study.
The most common complaint after treatment of a fracture of mandibular condyle is persistent restriction of mouth opening ${ }^{19}$. This is avoided in the present study as it showed significant difference between preoperative and at 12 month. As, an adequate physiotherapy and rehabilitation in the recovery period assess in the change amount of mouth opening. Physiotherapy in the recovery period is considered as a one of the main steps of closed reduction protocol of subcondylar fracture treatment, so that a mouth opening as large as possible can be achieved..

In the current study, the decrease in the ramus height at 12 months compared with pretreatment was not significant. This could be explained by the reduced displacement of the fractured condyle preventing the ramus to be pulled up by the muscles attached to the mandible as can be in case of a high condylar fracture, undisplaced fracture, or a greenstick fracture. This is in the same side of Eckelt et al ${ }^{20}$.

Finally, it can be concluded that conservative treatment is favorable protocol for treatment of unilateral subcondylar fractures of the mandible with no malocclusion. Adequate mouth opening, minimal displacement of condyle, and minimal ramus height shortening are other factors to be considered before opting for a closed treatment. Patients should be followed up for a long period to assess any worsening of mandibular function.

\section{REFERENCES}

1. Haug RH, Prather J, Indresano T. An epidemiologic survey of facial fractures and concomitant injuries. J Oral Maxillofac Surg 1990 ;48:926-32.

2. EllisE3 ${ }^{\text {rd }}$.MoosKF-AttarA,ArborA.Tenyearsofmandibular fractures: An analysis of 2137 cases. Oral surg oral med oral pathology.1985; 59(2):120-9.

3. Zachariades N, Mezitis M, Mourouzis C, et al: Fractures of the mandibular condyle: A review of 466 cases. Literature review, reflections on treatment and proposals. J CranioMaxillofacial Surg 34: 421, 2006. 
4. Jensen T, Jensen J, Nørholt SE, Dahl M, Lenk-Hansen L, Svensson P. Open reduction and rigid internal fixation of mandibular condylar fractures by an intraoral approach: a long-term follow-up study of 15 patients. J Oral Maxillofac Surg 2006;64(12):1771-1779

5. Zachariades N, Mezitis M, Mourouzis C, Papadakis D, Spanou A. Fractures of the mandibular condyle: a review of 466 cases.Literature review, reflections on treatment and proposals. J Craniomaxillofac Surg 2006;34(7):421-432

6. Landes CA,Day K,LipphardtR,SaderR.Closed versus open operative treatment of nondisplaced diacapitular (Class VI) fractures. J Oral Maxillofac Surg 2008;66(8):1586-1594

7. Ellis E III, Throckmorton GS. Treatment of mandibular condylar process fractures: biological considerations. J Oral Maxillofac Surg 2005; 63(1):115-134

8. Haug RH, BrandtMT.Closed reduction, open reduction, and endoscopic assistance: current thoughts on the management of mandibular condyle fractures. Plast Reconstr Surg 2007;120 (7, Suppl 2):90S-102S

9. Troulis MJ, Kaban LB. Endoscopic approach to the ramus/ condyle unit: clinical applications. J Oral Maxillofac Surg 2001;59(5):503-509

10. Zide MF, Kent JN. Indications for open reduction of mandibular condyle fractures. J Oral Maxillofac Surg 1983;41(2):89-98

11. Goss AN, Bosanquet AG. The arthroscopic appearance of acute temporomandibular joint trauma. J Oral Maxillofac Surg 1990; 48(8):780-783, discussion 784
12. Wood GD. Assessment of function following fracture of the mandible. Br Dent J 1980; 149(5):137-141.

13. Worsaae N, Thorn JJ: Surgical versus nonsurgical treatment of unilateral dislocated low subcondylar fractures: a clinical study of 52 cases. J Oral Maxillofac Surg 1994; 52: 353-360.

14. Hovinga J, Boering G, Stegenga B: Long term results of nonsurgical management of condylar fractures in children. Int J Oral Maxillofac Surg 1999; 28 : 429

15. Walker RV: Condylar fractures: nonsurgical management. J Oral Maxillofac Surg 52: 1185-1188, 1994.

16. Santler G, Kärcher H, Ruda C, Köle E. Fractures of the condylar process: surgical versus nonsurgical treatment. J Oral Maxillofac Surg 1999;57(4):392-397, discussion 397-398

17. MacLennan WD. Fractures of the mandibular condylar process. Br J Oral Surg 1969;7(1):31-39

18. Niezen ET, Bos RRM, de Bont LGM, Stegenga B, Dijkstra PU. Complaints related to mandibular function impairment after closed treatment of fractures of the mandibular condyle. Int J Oral Maxillofac Surg 2010;39 (7):660-665

19. Newman L.Aclinical evaluation of the long-term outcome of patients treated for bilateral fracture of the mandibular condyles. Br J Oral Maxillofac Surg 1998;36 (3):176-179

20. Eckelt U, Schneider M, Erasmus F, et al. Open versus closed treatment of fractures of the mandibular condylar process-a prospective randomized multi-centre study. J Craniomaxillofac Surg 2006;34(5):306-314 
مجلة أسيوط لطب الأسنان

النشر الرسمي لكلية طب الأسنان

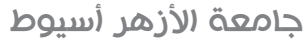

AADJ, Vol. 2, No. 1, APRIL (2019) - PP. 83:83

الملخص العربي

المدف من الدراسة:

الهدف من الدراسـة هو تقييم وظائف مفصل الفك بعد العلاج التحفظى لكسـور لقمة مفصل الفك من جانب واحد.

الموضوعات والأساليب:

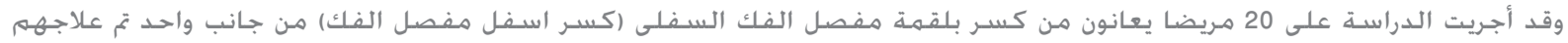

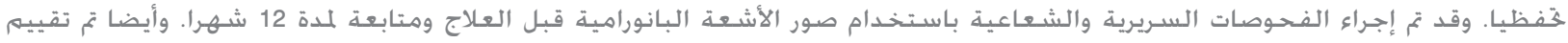

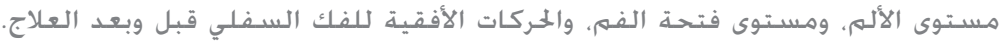

(النتائج:

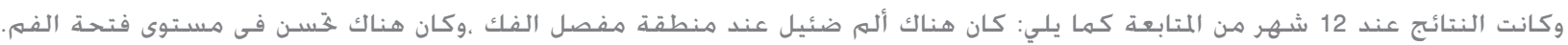

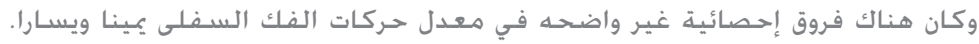

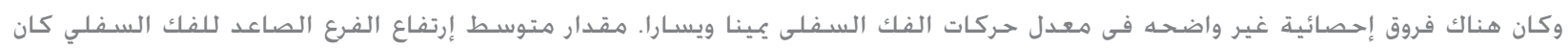
غير مهم عند التقييه الشعاعي ، فكانت قبل وعند و 12 شهرا بعد العلاج تساوى 1.51 م 0.45 و 1.47 + 0.48 ، على على التوالي.

الخلاصة:

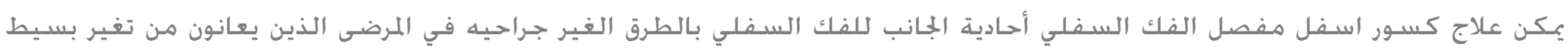

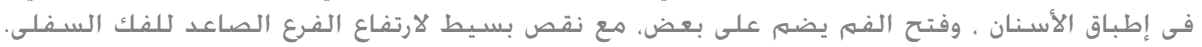

